\title{
KINETIC THEORY OF GASES IN GENERAL RELATIVITY THEORY
}

\author{
Jürgen Ehlers \\ Max-Planck-Institut für Physik und Astrophysik \\ Munchen
}

\section{CHAPTER I. INTRODUCTION}

The purpose of this lecture is, firstly, to describe the framework of the general-relativistic kinetic theory of gases and, secondly, to sketch some of the advances which have been made in this field during the last few years. Systematic expositions containing details and proofs can be found in references [1] - [6] and [29].

Some of the reasons for developing a general-relativistic kinetic theory of gases are the following. The traditional fluid description for the sources of gravitational fields does not seem to be appropriate in some cases of astrophysical interest such as stellar systems or the "galaxy-gas" of cosmology, since collisions are rare and the mean free paths are long. Also, a fluid description does not provide values for transport and reaction coefficients, whereas the less phenomenological kinetic theory does. Moreover, radiation (i lotons, neutrinos) can be described as a gas of zero-mass particles for some purposes, and only a relativistic version of kinetic theory can provide a unified treatment of such gases and ordinary gases. Also, relativistic kinetic theory helps clarifying controversial questions of relativistic thermodynamics. Finally, the relativistic version of kinetic theory is in some respects simpler and more transparent than its nonrelativistic predecessor; here as in other branches of Physics the unifying and simplifying power of the spacetime - geometrical point of view first put forward by $H$. Minkowski is clearly visible. 
CHAPTER II. REMARKS ABOUT GENERAL RELATIVITY THEORY

In Einstein's theory of gravitation spacetime, the arena of al1 physical processes, is assumed to be a four dimensional manifold which carries a pseudoriemannian metric. The metric tensor gab can locally be transformed to the Minkowski-form $g_{a b}=\operatorname{diag} .(1,1,1,-1)$. It determines the 1ight-cones, the distinction between time-1ike, space-1ike and nu11 (or light-like) vectors, it defines the causal structure of spacetime, and it establishes (part of) the connection between the mathematical formalism and Physics by providing definitions of (proper) times and distances. At the same time, the ten functions $g_{a b}\left(x^{c}\right)$ which, in the presence of inhomogeneous gravitational fields, cannot be transformed into constants by coordinate transformations in finite regions, act as potentials of the gravitational field. In a weak, quasistationary field, e.g., one has approximately

$$
d s^{2}=g_{a b} d x^{a} d x^{b}=d \vec{x}^{2}-\left(1+2 \frac{U}{c^{2}}\right) c^{2} d t^{2}
$$

where $U$ is the Newtonian gravitational potential, and in more general situations all ten $g_{a b}$ 's contribute to the field.

Just as in Newtonian theory the potential $U$ is related to the mass density $\rho$ of matter by Poisson's equation $\nabla^{2} U=4 \pi G_{p}$, so in Einstein's theory of gravitation the metric field $\mathrm{g}_{\mathrm{ab}}$ is coupled to matter by the field equation

$$
G^{a b}=T^{a b}
$$

Here, the Einstein tensor $\mathrm{G}^{\mathrm{ab}}$ is a symmetric second-rank tensor constructed from the $g_{a b}$ 's and their first and second derivatives, and $\mathrm{T}^{\mathrm{ab}}$ is the stress-energy-momentum tensor of all the matter (particles and non-gravitational fields) present. Here and in the sequel, the convention $G=\frac{1}{8 \pi}, c=1$ is used; later we shall a1so 
put $k$ (Boltzman's constant) $=1$.

Equation ( 1 ) implies the energy-momentum balance equation

$$
\mathrm{T}^{\mathrm{ab}} ; \mathrm{b}=0
$$

in which ( $; b$ denotes covariant differentiation with respect to $x^{b}$. In a gravitational field, eq. (2) is no longer a local

conservation law, but expresses the response of matter to gravity; it restricts (and in simple cases determines) the motion of bodies. As will be indicated later, eq. (2) can be derived from simpler assumptions in kinetic theory, independently of the field equation (1).

To solve Einstein's field equation (1) means, apart from specifying a manifold which serves as the domain for the tensors $\mathrm{g}_{\mathrm{ab}}, \mathrm{T}^{\mathrm{ab}}$ etc., to choose a physically reasonable model of matter which specifies the form of $\mathrm{T}^{\mathrm{ab}}$ in terms of matter or field variables (and of $g_{a b}$, and then to find values for the metric field and the matter variables which satisfy the ten coupled, quasilinear (but nonlinear:) differential equations (1), possibly in conjunction with further, non-gravitationa1, laws describing the sources. In general, neither the left-hand side nor the right-hand side of eq. (1) can be considered as given; one is faced with the problem of finding "simultaneously" all the quantities $g_{a b}, T^{a b}$ etc. such that they satisfy eq. (1) "selfconsistently".

In macroscopic applications of general relativity theory the standard model of matter has been the perfect fluid, given by its energy density $\mu$, its (isotropic) pressure $p$, and its 4-velocity $u^{a}$ (a timelike unit vector tangent to the streamlines); for it

$$
T^{a b}=\mu u^{a} u^{b}+p\left(g^{a b}+u^{a} u^{b}\right)
$$

In this case, eq. (2) is equivalent to a continuity equation for $\mu$ and a generalized Euler equation for $u^{a}$. Just as in Newtonian theory the system of equations (1), (3) is underdetermined; the simplest way 
to obtain a system such that Cauchy initial data uniquely determine the future evolution is to add a relation

$$
p=f(\mu)
$$

between pressure and density.

This model of matter is a very special one. Thermal phenomena are neglected in (3) and (4); in particular, no transport phenomena are taken into account. Although these drawbacks can be removed partly at the phenomenological level, the choice of non-equilibrium equations remains a matter of guesswork, and no transport coefficients are given. Moreover, if the matter of interest is radiation, a description like that in eq. (3), even if more or less correct, does not give sufficiently detailed information, since one would like to bring into the picture the spectrum of the radiation.

One simple way to improve the description of matter is to turn to kinetic theory, as will be done now. 
CHAPTER III. BASIC CONCEPTS AND LAWS OF RELATIVISTIC KINETIC THEORY

The theory to be outlined in this section was developed in smal1 pieces over a long period of time. The main steps have been taken by Jüttner (1911, 1928), Synge (1934), Walker (1936), Lichnerowicz and Marrot (1940), Chernikov (1960 - 1963), Tauber and Weinberg (1961), and Ehlers (1961).* Papers concerned with applications, approximation methods, special solutions etc. will be mentioned in section IV; no attempt is made, however, to give a complete list of references.

The assumptions on which the kinetic theory of gases is based are the following:

(a) The interactions between the particles constituting the gas can be divided into long range forces and weak, short range forces such that

(a) the long range forces can be accounted for in terms of a mean field generated collectively by the particles of the gas through macroscopic field equations, and

(a) 2 the short range forces can be taken into account in terms of (elastic or inelastic) point-collisions whose probability of occurence is governed by cross-sections taken from a special-relativistic scattering theory.

In accordance with this, it is assumed that

(b) between collisions, particles move like test particles in the mean field.

Finally, the usual assumption is made that

(c) the pattern of world-lines and collision events may be treated as a random structure whose (physically relevant) properties can be described by smooth expectation values.

See references [7], [8], [9], [10], [1], [11], [12], respectively. 
These assumptions are physically plausible for dilute gases. Their justification from first principles of many-particle dynamics is a formidable problem which is not attempted here; rather, we follow Boltzmann in formulating directly laws in a suitably defined one-particle phase space which seem reasonable under the above as sumptions.

As the only long range interaction we shall here take gravitation; electromagnetic fields can easily be included in an analogous way. As short range interactions we have in mind nongravitational interactions such as electromagnetic multipole forces or nuclear forces.

Let the gas consist of particles of proper mass $m(\geq 0)$. Between collisions, we have according to (b) geodesic motion, i.e.

$$
\frac{d x^{a}}{d v}=p^{a}, \frac{D p^{a}}{d v}=\frac{d p^{a}}{d v}+\Gamma_{b c}^{a} p^{b} p^{c}=0
$$

The parameter $v$ is chosen such that $\mathrm{p}^{\mathrm{a}}$ is the 4 -momentum, thus

$$
p_{a} p^{a}=-m^{2}
$$

D

$\overline{\mathrm{d} v}$ indicates the absolute derivative; the quantities

$$
\Gamma_{b c}^{a}=\frac{1}{2} g^{a d}\left(g_{d b, c}+g_{d c, b}-g_{b c, d}\right)
$$

form the components of the Riemannian connection associated with $\mathrm{g}_{\mathrm{ab}}$. Physically, these quantities are the relativistic analogues of the components of the gravitational field strength. Their non-tensorial character is (physically) due to the principle of equivalence. ${ }^{\text {The }}$ $g_{a b}$ and $\Gamma_{b c}^{a}$ represent the mean field according to assumption $(a)_{1}$.

+ The $\Gamma_{b c}^{a}$ 's form a good example of an object which is neither a tensor nor a spinor, but nevertheless of fundamental geometrical and physical importance. 
In nonrelativistic kinetic theory it is customary to represent the states of particles as points in a six-dimensional $(\vec{q}, \vec{p})$ phase space; these points move in the course of time. Such a description refers to a particular inertial frame of reference. If one passes from one inertial frame to another one, the phase-space description (of a particular gas state) changes in a simple and obvious manner. A similar description is still posibible in special relativity, and has in fact been employed (e.g., by Jütner). Here, the change connected with a change of the inertial frame is already more complicated due to the relativity of simultaneity. In general relativity, inertial frames (in finite domains) do not exist due to the very nature of gravitational fields*. Hence, the above description cannot be taken over without essential changes.

The best plan to overcome this difficulty is, not to use arbitrary, non-inertial frames of reference with a necessarily highly arbitrary splitting of spacetime into "space" and "time", but rather to look for the frame-independent meaning of the ordinary phase-space description, which can then be carried over to general relativity almost without change.

In geometric language the phase space description amounts to the following: In spacetime $X=\{\vec{x}, t\}$, the motion of a particle is represented as a worldiine $(\vec{x}[t], t)$. The instantaneous state of a particle with mass m can be specified by an event ( $x\left[t_{0}\right], t_{0}$ ) and a world-momentum $\left(\mathrm{m} \dot{\vec{x}}\left[\mathrm{t}_{0}\right], \mathrm{m}\right)$ at that event. The collection of all possible instantaneous states (for fixed $m$ ) is a seven-dimensional manifold $M$, the augmented phase space. A mean (or external) field defines in $M$ a family of curves, the phase flow; it represents all

\footnotetext{
* If inertial frames are to be identifiable locally by means of mechanical experiments or if unbounded matter distributions are considered (as in cosmology), this statement holds already in Newtonian theory.
} 
possible test particle orbits, "lifted" from $X$ to M. The sixdimensional manifold $\dot{M}$ of phase orbits (obtained from $M$ by identifying points contained in the same orbit) is the intrinsic object corresponding to the many $(\vec{q}, \vec{p})$ phase spaces associated with the various inertial frames.

One can assign a size to a tube $T$ of phase orbits by intersecting $T$ with a hypersurface $t=$ const, and forming the Lebesgue-measure $\int \mathrm{d}^{3} x \mathrm{~d}^{3} \mathrm{p}$ of that intersection, using inertial coordinates. According to Liouville's theorem, this size is independent both of the inertial frame used to compute it and of the instant $t$ defining the cross section. Thus, the Lebesgue measure defines a measure $\omega$ on $\dot{M}$.

The description just given applies to (special and) general relativity immediately. The augmented phase space is here given by

$$
M=\underset{X \varepsilon X}{U} P_{X}
$$

the union of all the mass-she11s $P_{x}=\left\{p^{a}: p_{a} p^{a}=-m^{2}\right\}$ belonging to the events* $x$ of $X$. (Coordinates in $M$ are $x^{a}, p^{\nu}$, where $a=1, \ldots$, 4 and $\nu=1,2,3, p^{4}$ is fixed by the mass shell condition (6).) The phase flow in $M$ is determined by equation (5). A measure on $\dot{M}$ is obtained as follows. First, form the product $\Omega=n \Lambda \pi$ of the Riemannian measure $n=\sqrt{-\operatorname{det}\left(\mathrm{g}_{\mathrm{ab}}\right)} \mathrm{d}^{4} \mathrm{x}$ of $\mathrm{X}$ with the measure $\pi=$ $\frac{d^{3} p}{p^{4}}$ (in orthonormal coordinates at $x$ ) of $p$, obtaining a measure on $M$ which can be shown to be invariant under the phase flow (5). Then, contract $^{\dagger} \Omega$ with the vector field ${ }^{+}$

* Px cannot be identified with Py for $x \neq y$ since parallel transport in $X$ is not integrable. $M$ is a fibre bundle over $X$, but not a cartesian product.

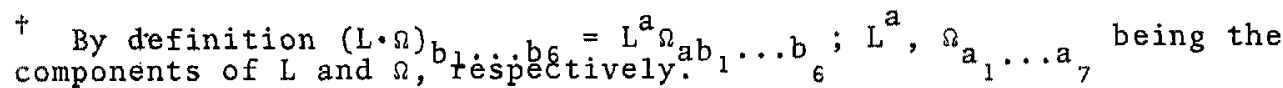
+ We follow the usage of modern differential geometry of identifying a vector with its directional derivative operator. 


$$
L=p^{a} \frac{\partial}{\partial x^{a}}-\Gamma_{b c}^{\nu} p^{b} p^{c} \frac{\partial}{\partial p^{v}}
$$

(on $M$ ) to obtain a six-form $\omega=L . \Omega$ which, again, can be shown to be invariant under the phase flow generated by the vector field (9). The measure of any region in $\dot{M}$, $i . e .$, any tube $T$ of phase orbits in $M$, is then defined (in strict analogy to the Newtonian case discussed above) as $f w$, taken over any cross section of $\mathrm{T}$.

The state or, rather, the history of an individual gas can be described by specifying those segments of phase orbits which are occupied by particles. It follows* from assumption (c) that the (average) number of occupied states (E phase orbit segments) intersecting a hypersurface $H$ of $M$ can be expressed as an integral

$$
N[H]=\int_{H} f \omega,
$$

where $f=f(x, p)$ is a non-negative, smooth, scalar function on $M$, called the (one particle) distribution function. Since w coincides for a local observer with the ordinary phase-element $d^{3} x d^{3} p$, $f$ has, for each such observer, the same meaning as the distribution function in nonrelativistic kinetic theory.

A collision $\left(x ; p, \bar{p} \rightarrow p^{\prime}, \bar{p}^{\prime}\right)$ at $x$ gives rise, in $M$, to two endpoints $(x, p),(x, \bar{p})$ of occupied phase orbit segments to be called annihilations, and two initial points, $\left(x, p^{\prime}\right),\left(x, \bar{p}^{\prime}\right)$, called creations. Counting the latter ones positively, the former ones negatively, one can easily deduce from (10) that the density of collisions in $M$ with respect to the measure $\Omega$ is given by

$$
L(f)=p^{a} \frac{\partial f}{\partial x^{a}}-\Gamma_{b c}^{\nu} p^{b} p^{c} \frac{\partial f}{\partial p^{\nu}} .
$$

Hence, the spacetime density of collisions at $x$ in which particles with 4 -momenta in $\pi$ are created, is given by $L(f) \pi$; this is the

* For a rigorous formulation and a proof, see [5a], [5b]. 
ordinary collision rate. *

The preceding remark implies: Absence of collisions or, more genera1ly, detailed balance between creations and annihilations (direct and inverse collisions) is expressed by

$$
L(f)=0 \text {, }
$$

the "collisionless" Boltzmann equation. (In the case of weak, quasistationary gravitational fields and slowly moving particles eqs. $(0),(7),(11)$, and (12) reduce to the well known gravitational

Vlasov equation

$$
\left.\frac{\partial f}{\partial t}+\frac{\vec{p}}{m} \cdot \frac{\partial f}{\partial x}-m \nabla U \cdot \frac{\partial f}{\partial \vec{p}}=0 .\right\}
$$

It is apparent from the meaning of $f$ that the moments

$$
\begin{aligned}
& N^{a}(x)=\int_{P_{x}} p^{a} f \pi, \\
& T^{a b}(x)=\int_{P_{x}} p^{a} p^{b} f \pi,
\end{aligned}
$$

represent currents in spacetime. $\mathrm{N}^{\mathrm{a}}$ is the particle 4-current density (also called numerical $f l u x$ ), and $T^{a b}$ is the kinetic stress energy momentum tensor of the gas described by $f$. In a similar way higher order moments can be defined.

Since $N^{a}$ is timelike, one can factor it into a non-negative scalar $\mathrm{n}$ and a timelike unit vector $\mathrm{u}^{\mathrm{a}}$;

$$
N^{a}=n u^{a} \cdot\left(u_{a} u^{a}=-1\right)
$$

An observer travelling with 4-velocity $\mathrm{u}^{\mathrm{a}}$ will observe no particle flux and will measure the particle density $n$. Hence, in accordance with nonrelativistic terminology one might call $\mathrm{u}^{\mathrm{a}}$ the mean 4 -velocity of the gas, and $n$, the proper particle density.

* See, e.g., references [2], [4], [5]. 
Any tensor $\mathrm{T}^{\mathrm{ab}}$ constructed via eq. (14) can be decomposed uniquely as *

$$
\mathrm{T}^{\mathrm{ab}}=\bar{\mu} \overline{\mathrm{u}}^{\mathrm{a}} \overline{\mathrm{u}}^{\mathrm{b}}+\overline{\mathrm{p}}^{\mathrm{ab}}
$$

with

$$
\bar{\mu} \geq 0, \quad \bar{u}_{\mathrm{a}} \overline{\mathrm{u}}^{\mathrm{a}}=-1, \quad \overline{\mathrm{p}}_{\mathrm{ab}} \overline{\mathrm{u}}^{\mathrm{b}}=0
$$

An observer travelling with 4 -velocity $\overrightarrow{\mathrm{u}}$ would, consequently, find the gas to have a vanishing momentum density. Thus, $\bar{u}^{a}$ could also be considered to be "the" mean 4-velocity of the gas. In general, however, $\bar{u}^{a} \neq u^{a}$. The physical reason for this is, of course, the velocity-dependence of the (relative) inertial mass of a particle. For clarity, $\mathbf{u}^{a}$ is called the kinematical mean 4-velocity, and $\bar{u}^{a}$ is called the dynamical mean 4-velocity of the gas (Synge 1956, [13]). (For a multicomponent gas the ambiguity in the choice of a mean 4-velocity is even greater; to avoid confusion, it is necessary to define precisely which mean velocity is used in a particular context.)

With respect to any mean 4 -velocity $\mathrm{u}^{\mathrm{a}}, \mathrm{T}^{\mathrm{ab}}$ can be uniquely decomposed according to ${ }^{\dagger}$

$$
\left.T^{a b}=\mu u^{a} u^{b}+p\left(g^{a b}+u^{a} u^{b}\right)+2 u^{(a} q^{b}\right)+\pi^{a b}
$$

with

$$
\mu \geq 0, u_{a} u^{a}=-1, u_{a} q^{a}=0, \pi_{a b} u^{b}=0, \pi_{a}^{a}=0
$$

The quantities $\mu, p, q^{a}, \pi^{a b}$ represent the energy density, mean kinetic pressure, energy current density, and shear viscosity with respect to $\mathrm{u}^{\mathrm{a}}$, respectively. We shall henceforth choose the $\mathrm{u}^{\mathrm{a}}$ in (18) to be the kinematical mean 4-velocity; then $\mathrm{q}^{\mathrm{a}}$ is also called

* Synge, [13], see also [5a], [5b].

+ By definition, $u^{\left(a{ }^{b}\right)}=\frac{1}{2}\left(u^{a} q^{b}+u^{b} q^{a}\right)$. 
the heat flux. $q^{a}=0$ if and only if $\bar{u}^{a}=u^{a}$; this property serves to define adiabatic processes.

Consider a spatially bounded gas, such as a gas enclosed in a container or (in good approximation) a star. An "instant of time" is represented in relativity as a spacelike hypersurface $G$ in $X$. $G$ defines a hypersurface $\hat{G}=\left\{(x, p): x \in G, p_{\varepsilon} P_{x}\right\}$ in the augmented phase space $M$. The entropy of the gas at the instant $G$ is defined to be

$$
S[G, f]=\underset{\hat{G}}{-\int_{f}} \mathbf{f} \log f \omega .
$$

This expression is the straightforward generalisation of the corresponding one in the nonrelativistic theory. It can be motivated either by adapting Boltzmann's counting procedure to the relativistic setting*; or by using a quantum model of a gas, starting from the definition $S=-\operatorname{trace}(W \log W)$ of its entropy in terms of its statistical operator $W$, and re-expressing that by means of correspondence arguments in terms of classical quantities ${ }^{\dagger}$. (The second procedure gives, of course, also the expressions appropriate to Bose or Fermi gases, both of which reduce essentially to (20) in the nondegenerate limit.)

If the form of the measure $w$ (which has been described above) is taken into account, it follows that (20) can be rewritten in the form

$$
S[G, f]=\int S_{G}^{a} \sigma_{a}
$$

where

$$
S^{a}(x)=-\int_{x} p^{a} f \log f \pi
$$

is a vector field in $X$ and $\sigma_{a}$ is the standard hypersurface element of G. $S^{a}$ is called the entropy flux of the gas.

\footnotetext{
See, e.g., references [7a], [14].

+ See reference [5a].
} 
In order to obtain a time evolution equation for $f$ one can carry over to relativity Boltzmann's collision hypothesis. Considering again a simple gas with elastic binary collisions only - other cases may be treated similarly - and remembering assumption (a) ${ }_{2}$ and the meaning of $L(f)$, one gets the Boltzmann equation ([1], [10], [11], [12])

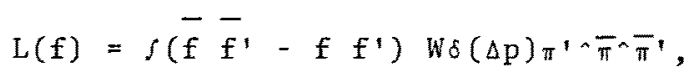

where the usual abbreviations $f^{\prime}=f\left(x, p^{\prime}\right)$ etc. have been employed and the nonnegative Lorentz invariant function $W\left(p, p^{\prime}, \bar{p}, \bar{p}^{\prime}\right)$ is related to the differential scattering cross section $\sigma(E, \theta)$ by (see refs. [3], [5], [32IV])

$$
W \delta(\Delta p) \bar{\pi}^{\wedge} \bar{\pi}^{r}=E\left(\left(\frac{E}{2}\right)^{2}-\mathrm{m}^{2}\right)^{\frac{1}{2}} \sigma(E, \theta) \mathrm{d} \bar{\Omega} .
$$

$\left(E=\left[-\left(p+p^{\prime}\right)^{2}\right]^{\frac{1}{2}}\right.$ is the total CM-energy, $\theta$ the CM scattering angle, and $\mathrm{d} \bar{\Omega}$ is a solid angle which refers to the direction of $\bar{p}$ in the CM frame of collision $\left[p, p^{\prime}+\bar{p}, \bar{p}^{\prime}\right]$.) The factor $\delta(\Delta p)=$ $\delta\left(\bar{p}+\bar{p}^{\prime}-p-p^{\prime}\right)$ accounts for conservation of 4 -momentum during collisions.

Whereas the left-hand side of eq. (23) is essentially generalrelativistic (see eq. (11)), the right-hand side is essentially special-relativistic; this conforms to the assumptions (a) and (a) stated above.

The Boltzmann equation implies the particle conservation law

$$
\mathrm{N}^{\mathrm{a}} ; \mathrm{a}=0
$$

and the 4-momentum balance equation (2), as is seen by differentiating covariantly eqs. (13) and (14) and using eq. (23). Similarly, differentiation of the entropy flux (eq. (22)) and use of eq. (23) leads to 


$$
\mathrm{s}^{\mathrm{a}} ; \mathrm{a} \geq 0
$$

the relativistic version of Clausius's inequality (H-theorem) [11], [12]. The quantity on the left-hand side of (26) is the (invariant) entropy production rate.

The last inequality can be used to motivate the definition of local equilibrium distributions as those distributions which, at an event $x$, have a vanishing entropy production rate. Using again the Boltzmann equation one can show*, as in nonrelativistic theory, that $f$ has the stated property if and only if $\log f$ is an additive collision invariant. This in turn implies that $f$ must have the form*

$$
f=e^{\alpha}+\frac{u_{a} p^{a}}{T},
$$

where $T>0$, and $u^{a}$ is a timelike unit vector ${ }^{\dagger}$. Eq. (27) gives the relativistic analog of the Maxwel1-Boltzmann distribution, first derived by probabilistic arguments by Jüttner (ref. [7a]). If (27) is combined with egs. (13), (14), and (22), then equations (15), (3) and $S^{a}=s^{a}$ follow, with well-determined functions $n(\alpha, T), \mu(\alpha, T)$, $p(\alpha, T), s(\alpha, T)$, together with standard thermodynamic relations which identify $T$ as the (absolute) temperature, and $\alpha=\frac{k}{T}+$ const. where $k$ is the chemical potential.

Global equilibrium in a spacetime domain D requires $f$ to have the form (27), with functions $\alpha(x), T(x), u^{a}(x)$ such that the Boltzmann equation holds. It turns out that this is true precisely if $\alpha=$ const. and

\footnotetext{
* For the most careful treatment of these points, see ref. [2a]. + If the right hand side of eq. (23) is modified so as to account for the Pauli principle or stimulated scattering, the analogous reasoning leads to the relativistic Fermi-Dirac and Bose-Einstein distributions, respectively ( [11]; see a1so [4], [5]).
} 


$$
\left(\frac{u_{a}}{T}\right) ; b+\left(\frac{u_{b}}{T}\right) ; a \quad \propto \quad\left\{\begin{array}{l}
0 \text { if } m>0 \\
g_{a b} \text { if } m=0
\end{array}\right.
$$

This means that $\frac{u^{a}}{T}=\xi^{a}$ must generate a group of congruent (if $m>0$ ) or conformal (if $\mathrm{m}=0$ ) mappings $\mathrm{x}^{\mathrm{a}}+\mathrm{x}^{\mathrm{a}}+\varepsilon \xi^{\mathrm{a}}$ of spacetime into itself, and that $T$ (and also $k$ ) must vary in $D$ just 1 ike $\left(-\xi_{a} \xi^{a}\right)^{-1 / 2}$. That is, for particles with positive rest mass $m$ global equilibrium is possible only in a stationary spacetime*, and then in stationary coordinates the temperature varies according to Tolman's law

$$
\mathrm{T} \sqrt{-\mathrm{g}_{44}}=\text { const. }
$$

$\left(x^{4}=\right.$ time coordinate, $\left.g_{a b, 4}=0\right)$. This means that the temperature depends on the gravitational potential in such a way that the gravitational redshift of photons does not disturb the equilibrium set up by exchange of radiation. $\left(-g_{44} \approx c^{2}+2 U\right.$, see eq. (0). )

If $m=0$, equilibrium is compatible with certain nonstationary states of the gravitational field. An important example is provided by black body radiation in an isotropically expanding space; this is the current model for the well-known $3^{\circ} \mathrm{K}$ cosmic fireball radiation. In this case, eq. (28) says that the radiation temperature drops like the inverse of the "world radius".

The fundamental equations for a gravitating gas (according to kinetic theory) are the Einstein field equation (1) with a source term as given by eq. (14), coupled with the Boltzmann equation (23). (Generalisations to gas mixtures, or to Fermion or Boson gases require obvious modifications.) Since both equations seperately imply eq. (2), it appears that they are compatible, and that the

* In nonrelativistic kinetic theory, distributions without entropy production are possible even in some non-stationary fields, as shown already by Boltzmann (1876). This is related to the question of bulk viscosity discussed briefly in section IV. 
Cauchy initial value problem for the system (1), (23) has a unique solution for "reasonable" initial data. Corresponding theorems (local existence, global uniqueness, and continuous dependence of the solutions on the initial data) have, in fact, been established recently for the collisionless case (see refs. [15a], [15b]), and the general case has essentially also been solved*. These rather deep results show that the kinetic theory model of a gravitating gas is mathematically consistent. The (local) stability of the solutions under small changes of the initial data, combined with Bichteler's result (see [16]) that exponentially bounded initial distributions (i.e., $|f(x, p)| \leq b(x) e^{\beta} a^{a}$ for some $\left.b, \beta_{a}\right)$ remain exponentially bounded for a finite time, lend some credibility to such formal approximation methods as those sketched in section IV.

* Private communication from Professor Y. Choquet-Bruhat. 
CHAPTER IV. REMARKS ABOUT SPECIAL SOLUTIONS AND APPROXIMATION METHODS FOR NON-EQUILIBRIUM SITUATIONS

a. No exact solutions of the relativistic Boltzmann equation (23), apart from the equilibrium solutions described above, are known if collisions are included (i.e., $W \neq 0$ ). In the collisionless case, eq. (23) is equivalent to the statement that the distribution function $f(x, p)$ is a first integral of the geodesic equation (5), and since many spacetime models have symmetries which give rise to such first integrals, several solutions of eq. (12) are known. If, e.g., $\xi^{a}(x)$ is a Killing vector ( of isometries), then the function $\xi_{a}(x) p^{a}$ on $M$ is a first integral of eq. (5), whence any positive function of it is a possible collisionless distribution function, and a corresponding remark applies if one has several Killing vectors. (For massless particles, conformal Killing vectors can also be used.) These integrals correspond to the energy, momentum and angular momentum integrals in fields with corresponding symmetries.

The preceding remarks apply in particular to static, spherically symmetric spacetimes, and have been used to compute the general solution of eq. (12) in such spacetimes which is invariant under the fu1l, four dimensional symmetry group* (SO[3]XR). The result can be used to compute $\mathrm{T}^{\mathrm{ab}}$ - eq. (14) - and to set up the Einstein equation (1). In this way, several solutions of the equations (1), (12) which provide models of relativistic star clusters have been constructed and have been used to estimate the quasistatic evolution of such objects (see references [17],[18]). Also, the stability of such systems against radial perturbations has been studied in a series of

\footnotetext{
* The action of any isometry group of a spacetime $X$ can easily be extended to the phase space $M$; thus it is meaningful to speak of the invariance of $f$ with respect to such a group.
} 
beautiful papers (references [19], [20]), and the results so far obtained indicate strongly that such clusters become unstable and collapse rapidly as soon as their central redshift exceeds a value of about 0.5 , a result which is of interest in connection with a quasar model proposed by Hoyle and Fowler.

Nonstationary solutions of eqs, (1), (12) have been found in connection with cosmological considerations. In particular, it has been established that if a solution has a locally rotationally symmetric distribution function with respect to some mean fourvelocity field, then, the mean motion is shear-free and either volume preserving or irrotional; and if it is not volume preserving, the metric must be of the Robertson-Walker type, i.e., it must correspond to a homogeneous and isotropic mode1 universe (refs. [21], [22]). In this case, the first integral on which the distribution function depends is not a linear one associated with a Killing vector - as in the static models - but is quadratic and of the form $\left(\xi_{\mathrm{a}} \xi_{\mathrm{b}}-\xi_{\mathrm{c}} \xi^{\mathrm{c}} \mathrm{g}_{\mathrm{ab}}\right) \mathrm{p}^{\mathrm{a}} \mathrm{p}$, where $\xi^{\mathrm{a}}$ is the conformal Killing vector associated with the isotropic expansion of the universe. (Similar quadratic integrals occur in the corresponding Newtonian solutions, see $r \in f$. [23]).

For further applications of kinetic theory to cosmology see references [4], [24], [25], and for some more solutions of eqs. (1), (12) see reference [26].

b. In order to describe non-equilibrium situations one has to resort to approximation methods. Restricting attention to nearequilibrium cases, one can write the actual distribution function $f$ as a "sma11" perturbation,

$$
f=e^{\alpha+\beta_{a p} p^{a}}(1+g)=f^{(0)}(1+g),
$$

of a local equilibriun distribution with parameters $\alpha(x), \beta_{a}(x)$ 
whose spacetime variation is to be determined from eq. (23) in conjunction with the small perturbation term $g(x, p)$.

As in nonrelativistic theory one can verify by means of eqs. (22), (13), (14), (18) that the equation of state $\mu=\mu(s, n)$, which relates the equilibrium values of energy density $\mu$, entropy density $s$ and particle density $n$, remains valid to first order in $g$ for a near-equilibrium distribution (30), if the mean velocity is taken to be $u^{a}{ }_{\propto \beta}{ }^{a}$ and $\mu, s$ and $n$ are defined, respectively, by eqs. (18), $s=-u_{a} s^{a}$, and $n=-u_{a} N^{a}$. Similarly one obtains that, to first order in $g$, the entropy flux relative to the mean motion, $s^{a}=s^{a}-s^{a}$, is related to the diffusion flux $i^{a}=N^{a}-n u^{a}$ and the heat flux $q^{a}$ (defined through eqs. [18], [19]) by $s^{a}=\beta q^{a}-(1+\alpha) i^{a}$. Hence, if one matches the parameters $\alpha, \beta^{a}$ in (30) to the actual distribution function $f$ by requiring $i^{a}=0$, one has the standard thermodynamic relation $\mathrm{s}^{\mathrm{a}}=\mathrm{Bq}^{\mathrm{a}}$. Combining these thermodynamic relations with the conservation laws (2) and (25) and using the Gibbs equation

$$
\mathrm{d} \mu=\mathrm{T} d s+\frac{\mu+\mathrm{p}_{\ell}-\mathrm{s}}{\mathrm{n}} \mathrm{dn}
$$

to define a temperature $\mathrm{T}$ and a thermodynamic pressure $\mathrm{p}_{0}$, one obtains the expression

$$
S_{; a}^{a}=-\frac{1}{T}\left\{\pi \theta+\pi_{a b} \sigma^{a b}+q^{a}\left(\frac{T, a}{T}+\dot{u}_{a}\right)\right\} \geq 0
$$

for the entropy production rate. Here the kinematical quantities $\sigma_{a b}, \theta$ and $\dot{u}_{a}$ are the shear velocity, the expansion rate, and the four-acceleration of the mean flow, defined by

$$
\left.\begin{array}{l}
u_{(a ; b)}=\sigma_{a b}+\frac{1}{3} \ominus\left(g_{a b}+u_{a} u_{b}\right), \\
a_{a b} u^{b}=0, \quad \sigma_{a}^{a}=0, \quad \dot{u}_{a}=u_{a ; b} u^{b},
\end{array}\right\}
$$


and

$$
\pi=p-p_{0}
$$

is the difference between the total kinetic pressure of eq. (18) and the thermodynamic pressure of eq. (31).

Al1 this follows standard lines of reasoning of nonrelativistic kinetic theory, and shows that the passage from kinetic theory to phenomenological thermo-hydrodynamics can be performed at the relativistic level as easily as in the standard theory, and this also holds for gas mixtures with diffusion and reactions (references [5b] and $[32 \mathrm{I}]$.

Equation (32) suggests the transport equations

$$
\left.\begin{array}{rl}
\pi_{a b} & =2 n \sigma_{a b}, \\
\pi & =-\xi \theta, \\
q_{a} & =-\lambda\left(\delta_{a}^{b}+u_{a} u^{b}\right)\left(T, b+T \dot{u}_{b}\right),
\end{array}\right\}
$$

with non-negative coefficients, $\eta, \xi, \lambda$. Specifically relativistic terms appear in the heat conduction law only. The acceleration term produces, in an equilibrium state, precisely the temperature variation which has been discussed on page 15 and which is, as we now see, needed to prevent heat from "falling" in a gravitational field, (Equations (35) have been proposed long ago, see ref. [27].)

Instead of guessing equations (35) on the basis of (32) one should, of course, derive them from the Boltzmann equation (23). Two classical methods offer themselves, the Chapman-Enskog method and Grad's, method of moments. Both these methods have, in fact, been adapted to relativity; as will be described briefly now.

The Chapman-Enskog method has been adapted to relativity by Israel (reference [6a]) and, in a mathematically more complete form, by Marle (reference [2b]). The method consists of replacing $W$ in 
equation (23) by $\frac{1}{\varepsilon}$, expanding $g$ in equation (30) in a power series $g=\sum_{n=1}^{\infty} \varepsilon^{n}(n)$, decomposing the Liouville operator (9)

$$
\begin{aligned}
L=p^{a} \nabla_{a} & =\underbrace{-u_{b} p^{b} u^{a} \nabla_{a}}_{D}+\underbrace{\left(\delta_{b}^{a}+u_{b} u^{a}\right) p^{b} \nabla_{a}}_{\nabla} \\
& =\underbrace{}_{a}=\frac{\partial}{\partial x^{a}}-\Gamma_{a b}^{\gamma} p_{\partial \frac{\partial}{p} \gamma}^{b})
\end{aligned}
$$

into a "time derivative" $D$ and a "spatial derivative" $\nabla$ (both operating in $M$ ), and to solve the resulting equation successively for each power of $E$ after elimination of $\dot{n}, \dot{\mu}$ and $\dot{u} \dot{a}\left(\dot{n}=n, a u^{a}\right.$ etc.) by means of the conservation laws (25), (2). In this procedure the variables $n, \mu, u_{a}$ have to be defined uniquely in terms of the "correct" distribution function $f$ by means of matching conditions, e.g., those of Landau-Lifschitz which require

$$
\left.\begin{array}{c}
\stackrel{\circ}{T^{a}}{ }_{b} u^{b}=T_{b}^{a} u^{b}=-\mu u^{a}, \\
\stackrel{\circ}{N}^{a} u_{a}=N^{a} u_{a}=-n .
\end{array}\right\}
$$

Here, $\stackrel{\circ}{N}^{a}, N^{a}$ are the currents (13) formed with $f(0), f$, respectively, etc. The result of this procedure in first order are the transport equations (35). To obtain the coefficients $\eta, \xi$ and $\lambda$, one has to solve inhomogeneous Fredholm integral equations. This has been done for "Maxwellian particles", defined (in relativity) by having a cross section of the seperable form $\sigma(E, \theta) \propto\left(\frac{E}{m}\right)^{-2}\left(\left[\frac{E}{2}\right]^{2}-1\right)^{-1 / 2} \Gamma(\theta)$, and for moderately relativistic temperatures (say, $\frac{T}{m} \leq 10^{-2}$ ), by Israel ( $\xi$ and $\lambda$, see [6a]) and by de Groot and van Leeuven $(\xi, \lambda$ and $\eta$, see $[32, V, V I])$. The last-mentioned authors extended these laborious calculations also to non-reacting mixtures of isobaric Maxwellian particles and established the validity of Onsager reciprocity relations for such systems (references [32,II,V]). Relativistic corrections to the transport coefficients, all of order $\frac{I}{\mathrm{~m}}$, have been 
worked out explicitly. An interesting result of these calculations is that $\xi$, the bulk viscosity coefficient, is positive, in contrast to the corresponding nonrelativistic result $\xi=0$ (for a simple gas of point particles). (In Israel's example $\xi$ is independent of $n$ and decreases for low temperature like $T^{3}$.) The reason for this deviation of the two theories can easily be seen to lie in the fact that the energy depends differently on the momentum in the two cases; $\xi=0$ happens to follow from the particular dependence $E=\frac{1}{2 \mathrm{~m}} \overrightarrow{\mathrm{p}}^{2}$ of nonrelativistic mechanics. The nonvanishing of $\xi$ "explains" the descrepancy between these two theories with respect to the existence of expanding local equilibrium flows (p.15): In relativity, even an isotropic expansion is connected with the production of entropy.

(For a critical discussion of this point and its bearing on cosmology, see $[28]$.

Whereas the method just sketched gives only normal solutions of the Boltzmann equation, the method of moments is also capable of describing the "anormal" relaxation towards equilibrium which cannot be described in terms of the "equilibrium variables" $n, \mu, u_{a}$ alone. This method has been modified according to relativistic requirements by Marle [2b] and by Anderson and Stewart (see [3] and volume 9 by Stewart of these "Lecture Notes", [29].)

The basic tool of this method is a complete orthogonal set of functions on the Hilbert space $L^{2}\left(P_{x}, f(0)_{\pi}\right)$, i.e., the space of real functions on the mass shell $\mathrm{P}_{\mathrm{x}}$ which are square integrable with respect to the measure $f(0) \pi$. The set is defined as follows. $H=1$, and $\stackrel{r}{H}^{a_{1}}, \ldots a_{r}=p^{a_{1}} \ldots P^{a_{r}}-\sum_{s=0}^{r-1} c_{b_{1}}^{a_{1} \ldots b_{s}} s_{h}^{b_{1} \ldots b_{s}} \quad(r=1,2, \ldots)$, with*

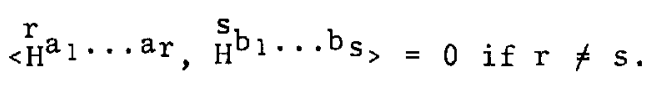

* The members of the set are the components of the tensors $\stackrel{r}{\mathrm{H}}$. For fixed $r$, these components are, in genera1, not orthogonal. 
(The C's are constants, and $<$, > denotes the inner product on the Hilbert space.) Such a set exists, is unique, the tensors $\mathrm{H}_{\mathrm{H}}{ }_{1}, \ldots a_{r}$ are symmetrical and trace free, and the set is complete. These functions form a relativistic analogue of the Hermite-Grad polynomials of $\mathrm{R}^{3}$, and reduce to them in a suitable limit*. (In contrast to the Hermite-Grad polynomials, the $\mathrm{H}^{\prime} \mathrm{s}$ cannot be derived from a generating function; they do not obey a Rodrigues relation.)

Assuming that $g$ from eq. (30) is a member of the Hilbert space one can expand it,

$$
g(x, p)=\sum_{n=1}^{\infty}{\stackrel{a}{b_{1}} \ldots b_{n}}_{(x)}{\stackrel{H}{b_{1}} \ldots b_{n}}_{(x, p)}
$$

The coefficients a... can be shown to be linear combinations of moments of $f$ of orders up to $n$.

Now, the Boltzmann equation (23) implies that

$$
\begin{aligned}
& \left(\delta p^{a} 1 \ldots p^{a_{n}} f \pi\right) ; a_{n}=\int p^{a} 1 \ldots p^{a_{n}-1} L(f) \pi
\end{aligned}
$$

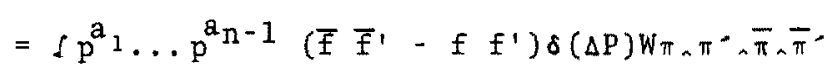

for $n=1,2, \ldots$, and conversely this infinite system of equations implies eq. (23). Inserting the expansion (39), the right hand side becomes a quadratic form in the $\mathrm{a} . .$. or, equivalently, in the moments of $f$, with coefficients expressible as integrals involving the $H^{\prime} s$. Hence, (40) represents a system of differential equations for the moments of $f$ which is equivalent to the Boltzmann equation. If one now truncates the series (39) after a few terms and linearises the truncated equations (40) in the a...'s one can obtain a tractable system of partial differential equations for the a...'s (or the moments), and these then define a moment-approximation of eq. (23). Keeping in (39) only the terms with $n=1$ and $n=2$, one gets the fourteen moment approximation (which corresponds to the

* For elegant proofs, see Marle [2b]. 
nonrelativistic thirteen moment approximation of Grad) which gives just sufficient information to derive again eqs. (35) (and, after stewart [29], more general equations for gas mixtures with reactions). In addition - and this is one of the principal advantages of this method compared with the first one - explicit integral representations are obtained for the transport coefficients ([3], [29], [6b]). The results concerning $\xi$ given above are confirmed and extended to arbitrary temperatures by this method. Moreover, this method permits to treat general, not only "normal" perturbations, and stewart has shown [29] that the behaviour of the $g$-dependent part of $\mathrm{T}^{\mathrm{ab}}$, the perturbed part of the stress tensor, is governed by a system of hyperbolic differential equations whose characteristics lie inside the light cone. For a simple Boltzmann gas, the maximal velocity of propagation of such disturbances (relative to the fluid) is $c\left(\frac{3}{5}\right) \mathbb{1}_{2}=0.8 \mathrm{c}$, which should be compared with the upper limit $\frac{c}{\sqrt{3}} \approx 0.58 \mathrm{c}$ for the sound velocity of such a gas [14]. Thus, an old paradox connected with eq. (35) 3 , the apparently acausal propagation of heat, has been resolved and has been shown to be due to an inadequate approximation. Extensions of this method to relativistic quantum gases are due to Stewart [29] and Israel and Vardalas [30].

As a last remark I wish to mention that a method which treats photons or neutrinos as a "gas" described by a distribution function, and which describes the medium with which this radiation interacts as a fluid - an approximation which is useful in astrophysical problems has been worked out in general relativity by Lindquist [31]; several applications of this theory of radiative transfer have been made, and more work along these lines is being carried out.

In conclusion it may be said that the basic conceptual and formal framework of relativistic kinetic theory is now well established, and 
that this new branch of statistical physics has proven to be a valuable tool of research which offers many possibilities for further investigation. 


\section{References:}

[1] N. A. CHERnikov: Acta Phys. Polon. 23, 629 (1963); 26, 1069 (1964), and earlier papers cited therein

[2] C. MARLE: (a) Ann. Inst. Henri Poincaré A 10, 67 (1969) (b) Ann. Inst. Henri Poincaré A 10, 127 (1969)

[3] J. L. ANDERSON: in Relativity, ed. M. Carmeli, S. J. Fickler, L. Witten (London: Plenum Press, 1970), p. 109

[4] R. K. SACHS AND J. EHLERS: in Astrophysics and Genera1 Relativity, ed. M. Chretien, S. Deser and J. Goldstein (New York: Gordon and Breach, 1971) Vol. 2, p. 331.

[5] J. EHLERS: (a) contribution to proceedings of the International School of Physics "Enrico Fermi" Course 47 (New York: Academic Press, 1971), p. I

(b) contribution to Relativistic Fluid Dynamics (Roma: Edizioni Cremonese, 1971), p. 301

[6] W. ISRAEL: (a) Journ. Math. Phys. 4, 1163 (1963) (b) contribution to Studies in Relativity (Oxford: Clarendon Press, to be published in 1972)

[7] F. JÜTTNeR: (a) Ann. Phys. 34, 856 (1911) (b) Ann. Phys. 35, 145 (1911) (c) Z. Physik 47, 542 (1928)

[8] J. L. SYNGE: Trans. Roy. Soc. Canada III 28, 127 (1934)

[9] A. G. WALKER: Proc. Edinburgh Math. Soc. 4, 238 (1936)

[10] A. LICHNEROWICZ AND R, MARROT: Comp. Rend. Acad. Sci. (France) 210, $759(1940)$

[11] G. E. TAUBER AND J. W. WEINBERG: Phys, Rev, 122, 1342 (1961)

[12] J. EHLERS: Abh, Akad. Wis5, Mainz (Jahrg. 1961), 791

[13] J. L. SYNGE: Relativity: The Special Theory (Amsterdam: North-Holland Publishing Co., 1956) 
[14] J. L. SYNGE: The Relativistic Gas (Amsterdam: NorthHolland Publishing Co., 1957)

[15] Y. CHOQUET-BRUHAT: (a) Journ. Math. Phys. 11, 3228 (1970)

(b) Ann. de 1'Institut Fourier (to appear in 1971)

[16] K. BICHTEleR: Commun. Math Phys. 4, 352 (1967)

[17] YA. B. ZEL'DOVICH AND M. A. PODURETS: Astr. Zh. $\underline{42}, 963$ (1965); english translation in Soviet Astron. AJ $\underline{9}, 742$ (1966)

[18] E, D. FACKERELL: (a) Ap. J, 153, 643 (1968) (b) Ap. J. 165, 489 (1971)

[19] J. R. IPSER AND K. S. THORNE: Ap. J. 154, 251 (1968)

[20] J. R. IPSER: (a) Ap. J. 156, 509 (1969) (b) Ap. J. 158, 17 (1969)

[21] J. EHLERS, P. GEREN AND R. K. SACHS: Journ. Math. Phys. 9, 1344 (1968)

[22] R. TRECIOKAS AND G. F. R. ELLIS: "Isotropic Solutions of the Einstein-Boltzmann Equations" preprint Univ. of Campridge, 1971 .

[23] J. EHLERS AND W. RIENSTRA: Ap. J. 155, 105 (1969)

[24] C. W. MISNER: Ap. J. 151, 431 (1968)

[25] R. A. MATZNER: Ap. J. 157, 1085 (1969)

[26] R. BEREZDIVIN AND R. K. SACHS: in Relativity ed. M. Carmeli, S. J. Fickler and L. Witten (London: Plenum Press, 1970), p. 125.

[27] C. ECKART: Phys, Rev, 58, 919 (1940)

[28] E. L. SCHÜCKING AND E. A. SPIEGEL: Comments Astrophys. Space Physics $\underline{2}, 121$ (1970)

[29] J. L. STEWART: Non-Equilibrium Relativistic Kinetic Theory, Lecture Notes in Physics (Berlin: SpringerVerlag, 1971), volume 9 
[30] W. ISRAEL AND J. N. VARDALAS: Nuovo Cimento Ser. I, 4 , 887 (1970)

[31] R. W. LINDQUIST: Annals of Physics 37, 487 (1966)

[32] S, R. DE GROOT, C. G. VAN WEERT, W. TH. HERMENS, AND W. A. VAN LEEUWEN: Physica 40, 257 (1968); 40, 581 (1969); 42, 309 (1969); W. A. Van Leeuwen and S. R. de Groot, Physica 51, 1; 16; 32 (1971). 